[Chem. Pharm. Bull.

35( 9 ) 3940-3942(1987) $]$

\title{
Dissolution Properties of Indomethacin from Coprecipitates with Water-Insoluble Glucan
}

\author{
Ken Masumoto, ${ }^{*, a}$ Akiyoshi Yoshida, ${ }^{a}$ Masami Hirayama, ${ }^{a}$ \\ SHIN'ICHI HaYashi, ${ }^{b}$ Yoshiharu MachIDA, ${ }^{c}$ \\ and TSUNEJI NAGAI ${ }^{c}$ \\ The Application and Development Division, ${ }^{a}$ the Research and Development Division ${ }^{b}$ \\ of Rohto Pharmaceutical Co., Ltd., Tatsumi Nishi 1-8-1, Ikuno-ku, Osaka 544, \\ Japan, and Faculty of Pharmaceutical Sciences, Hoshi University, ${ }^{c}$ \\ Ebara 2-4-41, Shinagawa-ku, Tokyo 142, Japan
}

(Received February 27, 1987)

\begin{abstract}
The dissolution properties of indomethacin (IMC) from coprecipitates with water-insoluble glucan (WIG) were investigated with a view to application of WIG to pharmaceutical preparations. WIG was produced by an enzyme present in the culture fluid of Streptococcus mutans. Coprecipitates were prepared by evaporation of the solvent from IMC solution in which WIG was suspended. The X-ray diffraction patterns indicated that the size of crystals of IMC was decreased by the coprecipitation. The dissolution rate of IMC from the coprecipitates was significantly greater than that from the physical mixture or intact IMC powder. These results indicate that WIG can improve the dissolution properties of IMC.
\end{abstract}

Keywords_-dissolution property; indomethacin; coprecipitate; water-insoluble glucan; Streptococcus mutans

In oral administration of poorly water-soluble drugs, the bioavailability depends mainly on the dissolution rate of the drug in the gastrointestinal fluid. ${ }^{1)}$ Therefore, if the dissolution rate. of such a drug were increased, the bioavailability might be improved, and consequently side effects might be reduced since a lower dose could be employed. From such a viewpoint, many studies have been carried out on means for enhancing the solubility and dissolution rate of poorly water-soluble drugs by the utilization of coprecipitates with polymers such as polyvinylpyrrolidone. ${ }^{2)}$

The authors have reported a method for the production of water-insoluble glucan $(\mathrm{WIG})^{3)}$ and have demonstrated the usefulness of WIG as a vehicle for directly compressed tablets ${ }^{4)}$ and sustained release tablets. ${ }^{5}$ In the present study, with a view to further application of WIG to pharmaceutical preparations, the effect of coprecipitation with WIG on the dissolution properties of a poorly water-soluble drug was investigated. Indomethacin, a practically water-insoluble non-steroidal antiinflammatory drug, was used as a model substance.

\section{Experimental}

Materials _ WIG obtained by the method described in the previous paper ${ }^{3)}$ and indomethacin (IMC) of JP XI grade were used after being passed through a 100-200 mesh sieve.

Preparation of Physical Mixture - A physical mixture of IMC with WIG in a $1: 3$ weight ratio was prepared by simple blending in a mortar.

Preparation of Coprecipitate-IMC was dissolved and WIG was suspended in $1: 1,1: 2$ and $1: 3$ weight ratios in ethanol at $50^{\circ} \mathrm{C}$. The solvent was removed in vacuo using a rotary evaporator at $50^{\circ} \mathrm{C}$, then the residue was dried in vacuo at room temperature for $24 \mathrm{~h}$, ground in a mortar and used after being passed through a $100-200$ mesh sieve. 
Powder X-Ray Diffraction Study_-Powder X-ray diffractometry was carried out using a diffractometer (Geigerflex model D-2, Rigaku Denki, Tokyo, Japan) with Ni-filtered, Cu- $K_{\alpha}$ radiation.

Dissolution Rate Study - The dissolution rate study was carried out by a beaker method. Sample powder, which corresponded to $25 \mathrm{mg}$ of IMC, was dispersed in $50 \mathrm{ml}$ of pH $6.0,1 / 15 \mathrm{M}$ phosphate buffer solution at $37^{\circ} \mathrm{C}$. After the addition of the powder, vigorous agitation was applied with a magnetic stirrer. At appropriate time intervals, $1 \mathrm{ml}$ of the solution was taken out with a $1 \mathrm{ml}$ transfer pipette and filtered with a membrane filter (TM-2 $0.45 \mu \mathrm{m}$, Toyo Roshi, Tokyo, Japan). The concentration of IMC in the filtrate was measured by the ultraviolet (UV) absorption method at $265 \mathrm{~nm}$ using a spectrophotometer (model UV-240, Shimadzu Corp., Kyoto, Japan) after dilution with $1 / 15 \mathrm{~m}$ phosphate buffer solution at $\mathrm{pH} 6.0$. WIG has no UV absorption at $265 \mathrm{~nm}$ and did not affect the measurement of IMC.

\section{Results and Discussion}

\section{Powder X-Ray Diffraction Study}

Powder X-ray diffraction patterns of IMC powder, WIG powder, physical mixture and coprecipitates are shown in Fig. 1. Several sharp diffraction peaks attributed to IMC crystals can be seen in the case of the physical mixture. However, those peaks were almost absent in the coprecipitates of all weight ratios, suggesting a decrease in the crystallinity of IMC as a result of the coprecipitation.

\section{Dissolution Study}

Dissolution profiles of IMC from the coprecipitates are shown in Fig. 2 in comparison with those from the physical mixture or intact IMC powder. The dissolution rates of IMC from the coprecipitates were significantly greater than that from IMC alone or the physical mixture. The dissolution rate of IMC from the coprecipitates increased as the ratio of WIG to

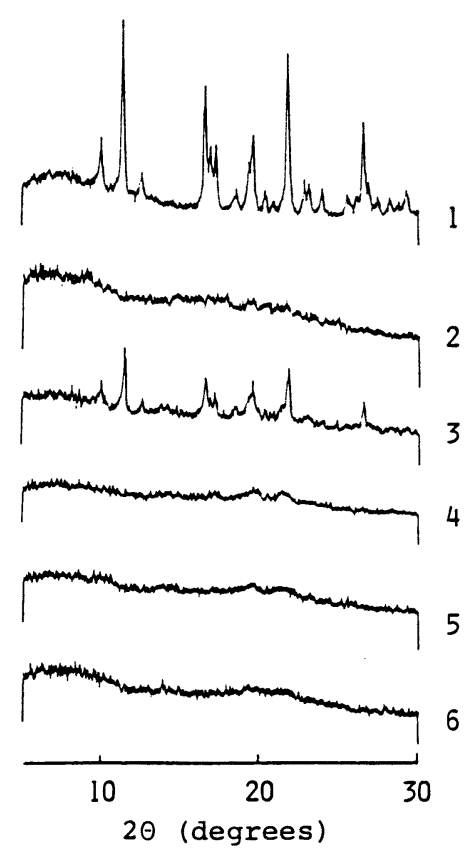

Fig. 1. Powder X-Ray Diffraction Patterns of IMC-WIG Coprecipitates in Different Ratios

1, IMC alone; 2, WIG alone; 3, physical mixture $(1: 3) ; 4$, coprecipitate $(1: 1) ; 5$, coprecipitate $(1: 2)$; 6 , coprecipitate $(1: 3)$.

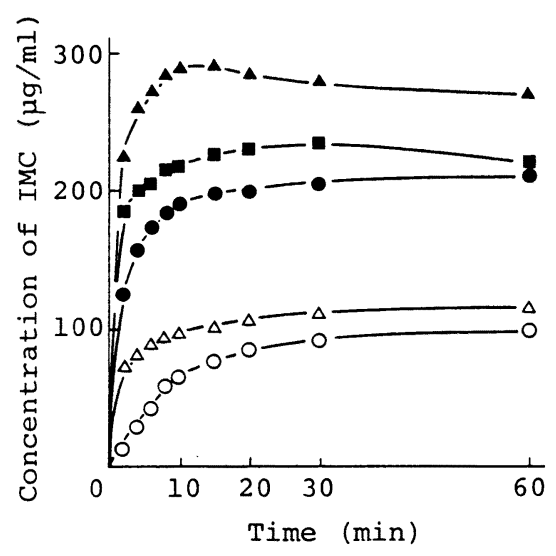

Fig. 2. Dissolution Profiles of IMC from Coprecipitates with WIG

○, IMC: $\mathrm{WIG}=1: 1$ coprecipitate; $\mathbf{\square}, 1: 2$ coprecipitate; $\Delta, 1: 3$ coprecipitate; $\triangle, 1: 3$ physical mixture; $\mathrm{O}$, IMC alone. Each point represents the mean of three determinations. 
IMC was increased. When the ratio of IMC to WIG was $1: 3$ or $1: 2$, the concentration of IMC reached a peak after 15 or $30 \mathrm{~min}$, respectively, then decreased gradually. Since the solubility of IMC in this medium at $37^{\circ} \mathrm{C}$ was $160.0 \mu \mathrm{g} / \mathrm{ml}$, supersaturation occurred in the initial stage of dissolution of the coprecipitate. IMC in the physical mixture dissolved more rapidly than IMC alone in the initial stage. The difference of dissolution of IMC between the physical mixture and IMC alone may be explained by the additive dispersion effect as demonstrated by Shah et al. ${ }^{6)}$

Thus, coprecipitation of IMC with WIG reduced the crystallinity of IMC, and the dissolution rate of IMC was enhanced. Therefore, the method of coprecipitation of poorly water-soluble drugs with WIG might be useful to improve the bioavailability and to reduce the side effects of such drugs.

\section{References and Notes}

1) A. J. Aguiar, J. KRC. Jr., A. W. Kinkel, and J. C. Samyn, J. Pharm. Sci., 56, 847 (1967).

2) K. Takayama, N. Nambu, and T. Nagai, Chem. Pharm. Bull., 28, 3304 (1980); idem, ibid., 29, 2718 (1981); H. Sekikawa, J. Fujiwara, T. Naganuma, M. Nakano, and T. Arita, ibid., 26, 3033 (1978); H. Sekikawa, M. Nakano, and T. Arita, ibid., 27, 1223 (1979); H. Sekikawa, N. Yagi, J. Sakuragi, K. Tanaka, M. Sakamoto, M. Itoh, M. Takada, and T. Arita, ibid., 30, 739 (1982).

3) K. Masumoto, K. Yamashita, A. Yoshida, S. Hayashi, Y. Machida, and T. Nagai, Chem. Pharm. Bull., 35, 3813 (1987).

4) K. Masumoto, N. Nambu, A. Yoshida, S. Hayashi, and T. Nagai, Chem. Pharm. Bull., 31, 209 (1983).

5) K. Masumoto, K. Matsumoto, A. Yoshida, S. Hayashi, N. Nambu, and T. Nagai, Chem. Pharm. Bull., 32,1055 (1984); idem, ibid., 32, 3720 (1984).

6) N. Shah, R. Pytelewski, H. Eisen, and C. I. Jarowski, J. Pharm. Sci., 63, 339 (1974). 